\title{
Using Personality Traits and Chronotype to Support Personalization and Feedback in a Sleep Health Behavior Change Support System
}

\author{
Holly Nguyen \\ Worcester Polytechnic Institute \\ hanguyen@wpi.edu
}

\author{
Carolina Ruiz \\ Worcester Polytechnic Institute \\ ruiz@wpi.edu
}

\author{
Vance Wilson \\ Worcester Polytechnic Institute \\ vwilson@wpi.edu
}

\author{
Diane Strong \\ Worcester Polytechnic Institute \\ dstrong@wpi.edu
}

\author{
Soussan Djamasbi \\ Worcester Polytechnic Institute \\ djamasbi@wpi.edu
}

\begin{abstract}
This paper addresses the issue of sleep deprivation among college students by proposing an innovative approach to personalizing a behavior change support system (BCSS) implemented within a smartphone sleep app. The proposed app extension uses individual personality and chronotype characteristics to support personalized feedback to users about their sleep patterns. Users' personality and chronotype are assessed using questionnaires, the results of which are used to personalize the content, timing and frequency of the app's feedback, which is designed to encourage healthier sleep behaviors.
\end{abstract}

\section{Introduction}

College students are notorious for their lack of sleep and irregular sleep patterns [1]. Although sleep may seem like a trivial and habitual activity, it actually fulfills a wide expanse of functions. Sleep deprivation can have serious health repercussions and can be detrimental to academic success [2].

Sleep deprivation is an issue that cannot be solved immediately because it requires substantive shifts in behavior. However, self-awareness and creation of goals to change unhealthy sleep patterns are good steps to begin this process.

This paper describes an approach to encouraging behavioral change in sleep by providing personalized feedback using a behavior change support system (BCSS) implemented within the SleepHealth smartphone app [3]. This app tracks users' total sleep time in comparison with their personal sleep goals, specified as a number of hours of sleep per night. A smartphone app is an effective tool for this purpose because most college students now use smartphones on a daily basis [4]. This high level of engagement with a smartphone will help our enhanced SleepHealth app to regularly encourage users to adhere to their sleep goals and promote self-awareness of their sleep patterns.

In this research, we are proposing an enhancement to the SleepHealth app to add system-personalized feedback. Engaging users in reviewing feedback about their own sleep patterns aims to promote consistent use of the app, leading to ongoing positive behavioral change. Our proposed personalized feedback will be delivered in the form of regular messages from the app. Our feedback design encompasses several design principles that are considered essential to the persuasive system design (PSD) model: praise, rewards, reminders, and suggestions [5].

Personalization - in this case, production of personalized content and services for SleepHealth users-is a separate PSD design principle [6]. Personalization in our research design will be developed based on users' personality and chronotype characteristics. Personalization of feedback should increase the likelihood that feedback will be relevant and engaging to users.

Recent personality research has linked sleep behavior to the Five-Factor Model (FFM) of personality, which encompasses five personality traits: conscientiousness, neuroticism, extraversion, agreeableness, and openness [7]. As explained later in this paper, individuals' personality characteristics can be used to predict the types of unhealthy sleep behavior they are most at risk for and the types of feedback that they are most receptive to.

Chronotype describes individuals' natural inclinations toward the times they are most alert or drowsy as well as their preferences for morning or evening activity [7]. Even a slight preference for morning or evening activity can impact sleep schedules. Thus, we anticipate chronotype will be an important factor to consider in developing personalized feedback to motivate healthier sleep patterns.

This research contributes by building on a recent stream of studies that apply PSD and BCSS theories to 
improvement of sleep health [5, 8, 9]. In addition to jointly investigating PSD personalization and feedbackrelated design principles through a BCSS implementation, we innovate further by incorporating individual characteristics of user personality and chronotype into our research design.

\section{Background}

\subsection{General Sleep Health}

Shifts in sleep behavior occur as individuals progress through adolescence and become young adults. According to the National Sleep Foundation, high school- and college-age individuals tend to adopt a later bedtime [1], and develop misalignment between their weekend and weekday sleep patterns [2]. This data is concerning in and of itself because it demonstrates an automatic shift toward unhealthy sleep behavior as length of sleep is reduced and patterns become more erratic. When these changing sleep patterns combine with lifestyle changes and social and academic pressures that accompany the move to a college environment, sleep deprivation can become a serious health problem. The National Sleep Foundation [10] observes that young adults need 8 to 10 hours of sleep each night but typically only get between 6.1 and 7.4 hours of sleep.

\subsection{Consequences of Sleep Deprivation}

Sleep deprivation can have serious health consequences, including higher risk for diseases, reduced cognitive function, and poor academic performance. Lack of sleep can negatively affect an individual's immune system, metabolism, inflammatory process, and cholesterol [11]. It can also impair memory formation, critical thinking skills [11] and sustained attention, all of which are necessary for academic success [12]. Erratic sleep patterns have been linked to reduced activation in cortical and subcortical rewardrelated brain regions [13]. This lack of neural activation can lead individuals to engage in reward-seeking behavior, which can result in poor decision-making and excessive risk taking [13]. For example, these risks include drowsy driving or increased alcohol use [2, 13].

\subsection{Sleep Deprivation Causes and Solutions}

Lack of sleep can be rooted in many causes. One factor is usage of electronic devices, such as video and gaming systems, before bedtime, which can reduce sleep quality (e.g., contribute to difficulties falling asleep, less restful sleep, and repeated awakenings) due to light from the device suppressing melatonin levels [2].

Another factor is lack of exercise. The results of a three-week study of 51 adolescents demonstrated that 30 minutes of regular exercise each weekday could provide longer total sleep time (TST), shorter sleep onset latency (SOL) and higher Rapid Eye Movement (REM) sleep latency [14].

Additionally, caffeine consumption can harm the length and quality of sleep. According to a Nature and Science of Sleep study, 34\% of 18-24 year olds consume energy drinks regularly [2], and the effects of caffeine consumption can last up to 7.5 hours after consumption [2]. Caffeine can be used to artificially reduce daytime sleepiness in order to remain awake. This may temporarily alleviate drowsiness when individuals are sleep-deprived [15], however, it does not resolve the underlying problem. Results of a survey of 844 undergraduate college students found that higher consumption of energy drinks is linked to lower GPAs (Grade Point Averages) as well as lower TST [16].

Reducing the use of electronics, increasing the amount of exercise, stopping caffeine consumption by $5 \mathrm{pm}$, and maintaining a regular sleep schedule are some effective methods to improve sleep health. However, there is often a dismissal of the need for better sleep health in the college culture, which makes it difficult to raise awareness of this issue.

\subsection{Personality, Chronotype, and Sleep}

Individual characteristics relating to personality and chronotype are important contributors to sleep patterns. Many studies have linked sleep behavior to personality traits comprising the FFM [17]. These five traits are defined in the following way [18].

- Conscientiousness is characterized by a tendency to be reliable, well-organized, and hardworking.

- Neuroticism reflects self-consciousness, insecurity, and tendency to worry.

- Extraversion describes behavior that is sociable, affectionate, cheerful, and assertive.

- Agreeableness is characterized by a tendency to be softhearted, sympathetic, and trusting.

- Openness is marked by originality, openmindedness, and creativity.

A study published by Behavioral Sleep Medicine found that lower scores for conscientiousness are linked to irregular sleep patterns due to lower levels of selfcontrol [1]. This finding is corroborated by a survey of 77 college freshmen that suggests a positive correlation between sleep quality and conscientiousness and a negative correlation between sleep quality and neuroticism [17]. The combination of high neuroticism 
and low conscientiousness is a key predictor of poor sleep [17].

A second type of individual characteristic that is important to sleep is chronotype, describing an individual's morning or evening preference. A propensity for morningness correlates with higher levels of conscientiousness and openness, and with lower levels of neuroticism [7]. This finding suggests that chronotype may also be an effective predictor of preference for various sleep patterns and, potentially, a useful factor in developing feedback to promote sleep health.

\subsection{Available Sleep Apps}

Mobile applications already exist to track an individual's sleep and allow the user to set a sleep duration goal. For example, the SleepBot app tracks total sleep time and sleep debt and provides a graphical display. Another sleep-tracking app, called Pillow, lets users set a sleep goal and view daily statistics, audio recordings of a user's sleep, and other factors such as eating late, on vacation, or reading a book. However, these apps do not engage the user through any personalized feedback.

Many of the sleep apps available in the market offer sleep-tracking functions and even can record a user's restlessness during sleep. But none of these apps seek to assess how personality and chronotype affect sleep and how these factors can be used to make the user experience more engaging and to encourage healthier sleep behaviors.

\subsection{Motivating Behavioral Change}

Our approach in this research follows the guidance of PSD and BCSS models [6, 19, 20]. These two theoretical models are highly compatible and synergistic [5]. As Oinas-Kukkonen ([19] p. 8) writes, "The Persuasive Systems Design model ... is the state of the art conceptualization for designing and developing BCSSs".

Our objective of motivating behavior change through use of personalization and feedback features in the SleepHealth app may be mapped to the BCSS Outcome/Change Design Matrix [19] as shown in Table 1. In this study, we focus only on the behavioral aspects of the matrix (i.e., compliance and behavior) rather than attitude.

We propose that personalization will primarily influence formation of healthy sleep behaviors, including initial compliance, and that feedback will primarily influence alteration and reinforcement of those behaviors. We expect personalization features will engage users, causing them to become more invested in the app as they perceive themselves taking initial actions of use [21].

Feedback will help users recall their target goals, provide documentation of their sleep history, and present new perspectives based on their own personality and chronotype characteristics. We anticipate this information will help users reflect upon and alter their behaviors and reinforce behavioral changes.

Table 1. Motivating behavior change through personalization and feedback

\begin{tabular}{|l|c|c|}
\hline & $\begin{array}{c}\text { C-Change } \\
\text { (Compliance) }\end{array}$ & $\begin{array}{c}\text { B-Change } \\
\text { (Behavior) }\end{array}$ \\
\hline $\begin{array}{l}\text { F-Outcome } \\
\text { (Formation) }\end{array}$ & $\begin{array}{c}\text { F/C } \\
\text { Personalization }\end{array}$ & $\begin{array}{c}\text { F/B } \\
\text { Personalization }\end{array}$ \\
\hline $\begin{array}{l}\text { A-Outcome } \\
\text { (Alteration) }\end{array}$ & $\begin{array}{c}\text { A/C } \\
\text { Feedback }\end{array}$ & $\begin{array}{c}\text { A/B } \\
\text { Feedback }\end{array}$ \\
\hline $\begin{array}{l}\text { R-Outcome } \\
\text { (Reinforcement) }\end{array}$ & $\begin{array}{c}\text { R/C } \\
\text { Feedback }\end{array}$ & $\begin{array}{c}\text { R/B } \\
\text { Feedback }\end{array}$ \\
\hline
\end{tabular}

\section{Methods and Approach}

\subsection{Measuring Personality and Chronotype}

Personality and chronotype are the individual characteristics we propose as appropriate for our objective of developing effective, personalized feedback relating to app users' sleep health. Both of these factors are assessed using existing questionnaires.

Personality characteristics are measured using the Big Five Personality Test [22], which consists of 50 questions. It provides a rating of low, medium or high for each of the five personality factors. Research has been conducted about the accuracy and validity of Big Five Inventories and whether they can be reliably used in research projects. Not only have many studies relied on the Big Five framework but research shows that these factors are enduring personality traits and "are found in different age, sex, race, and language groups" [23]. One such study demonstrates that even a 15-question Big Five Inventory, known as the BFI-S, is still a reliable instrument for measuring personality [24]. Therefore, the 50 question Big Five Personality Test that this research uses is a reliable and valid method to collect personality data.

Chronotype is measured using the Composite Scale of Morningness (CSM) [25]. The CSM is scored on a number range with a score below 22 indicating an evening type and a score above 44 indicating a morning type. Any person who receives a score between 22 and 
44 is not definitively a morning or evening type. However, any score above 22 and lower than 33 indicates a tendency towards being an evening type whereas a score above 33 but lower than 44 indicates a tendency towards being a morning type. The CSM was tested in another study for validity based on correlating actual sleep patterns and performance with scores to determine if the CSM provided a valid measurement [26]. This study validated the overall CSM scores, though another study found that the CSM scores across cultures fluctuated in terms of the setpoint for cutoff scores. However, this paper's proposed framework is not yet tackling transcultural research and is a future concern [27].

\subsection{Designing Personalized Feedback}

Different levels of these individual characteristics or specific combinations are likely to predict different potential problems in sleep patterns. Furthermore, different feedback is likely to work better for users with different characteristics in encouraging them to change their behaviors to healthier ones.

In section 4, we present our analysis and predictions of how individual personality and chronotype characteristics are likely to affect sleep patterns. Where problems are predicted, we also predict the feedback design most appropriate for those characteristics. By feedback design we mean feedback that is personalized by varying the content, timing, and frequency of feedback messages.

\subsection{Creating an Enhanced SleepHealth App}

Our plan for implementing our personalized feedback design starts with the SleepHealth app [3]. SleepHealth is a smartphone app with features for users to set a sleep goal of a certain number of hours each night, track sleep and wakening times, view sleep history in a graphical display, and track caffeine intake and exercise statistics.

We propose creating an enhanced version of the SleepHealth app that provides feedback personalized to each user based on individual personality and chronotype characteristics. We also intend to add messages about progress toward their individual sleep goals, a feature not currently in the app. In this manner, feedback will supplement simple tracking and viewing of a user's sleep behavior.

Our proposed design for the enhanced SleepHealth app is presented in Section 5 in the form of a concept prototype. A concept prototype is a high-level prototype that illustrates the vision with respect to functionality, design and operations. In our case, we provide screen shots of collecting chronotype data and providing personalized feedback as well as a general description of the prototype's design and functionality.

\section{Personalized Feedback Design}

In this section, we present our design for how personality and chronotype characteristics of users can be leveraged to increase the effectiveness of BCSS feedback in promoting and sustaining behavior change. We examine each individual characteristic and describe how it affects, or is likely to affect, sleep behaviors and how feedback should be tailored to promote healthier sleep behaviors for individuals with that characteristic.

The results of this analysis are shown in Table 2 , which presents our design for how feedback should be personalized depending on individual characteristics. The feedback design presented in Table 2 is relatively high level to show clearly the relationships between the individual characteristics and the goal of the feedback. Using this design, we will then generate a range of actual feedback messages for each identified personalization instance so that we have a variety of messages to send to users so as to avoid becoming repetitive and stale.

\subsection{Conscientiousness}

One of the most important personality factors to assess is conscientiousness, due to its correlation with better and longer sleep [1]. Individuals with low levels of conscientiousness are more susceptible to poor sleep behavior due to lack of self-control, which may play a role in weekend/weekday misalignment as well as a late bedtime. Due to these tendencies, the implementation of personalized feedback will vary depending on whether individuals score low or high for conscientiousness.

For those low in conscientiousness, their lack of self-control could result in their forgetting their sleep goal, which is why feedback for those users will occur twice per week. Additionally, an absence of willpower increases the likelihood of weekday/weekend misalignment, a situation where sleep patterns change erratically. Thus the second reminder message will be transmitted on Fridays.

Those who score high in conscientiousness are more goal-driven, which is why their feedback will be sent on Mondays and focus on their goal for the week. Also, those scoring high in conscientiousness tend to be organized, prepared and detail-oriented [28]. These characteristics make it more likely that a conscientious person will succeed academically. Therefore, their reminders will be targeted towards avoiding behavior that may increase short-term academic success, but can negatively impact long-term health and academics, such 
as carrying out all-night study sessions and excessive use of caffeine to do so. Not receiving any sleep for one night is detrimental to the regularity of one's sleep schedule. Also, excessive consumption of caffeine can negatively impact quality of sleep, as stated in Sec. 2.3.

\subsection{Neuroticism}

Another factor that is strongly correlated with sleep behavior is neuroticism. Highly neurotic individuals have less emotional stability, are more prone to impulses and, therefore, are more likely to be affected by stress [28]. High neuroticism has been correlated with poor sleep habits and poor sleep quality [7]. An individual with a high score in neuroticism is significantly more likely to already exhibit unhealthy sleep behavior. However, any harmful sleep patterns may manifest in different ways, including short sleep duration and long sleep duration, both of which have been associated with high neuroticism [18]. Highly neurotic individuals may respond to stress in different manners. Some may lean towards insomnia in the case where stress prevents sufficient sleep. Others may be on the other end of the scale where the extreme stress necessitates an exorbitant amount of sleep in order to cope.

Therefore, those scoring high in neuroticism will receive feedback focused on the underlying problem: controlling stress. Thus, reminders may include tips and references about mindfulness, resisting impulses, prioritization techniques, coping skills, and encouragement to exercise in order to reduce stress [7]. These reminders will occur twice per week to provide continual reinforcement about incorporating positive behaviors into daily life, which would positively affect sleep behavior. On the other hand, individuals with low neuroticism may not require any particular feedback or reminders based merely on their personality type.

\subsection{Extraversion}

A high score for extraversion indicates that the individual tends towards an outgoing personality and, therefore, is more likely to seek out social situations [28]. While higher extraversion is typically characterized by lower stress levels and shorter sleep latency, there is still the possibility for social situations to impact a highly extraverted individual's sleep patterns [18]. For example, highly extraverted individuals may be more likely to support their social needs by staying up late, especially on weekends. Hence, feedback encouraging regular sleep hours during weekends is likely to help this group of people.

For a low score in extraversion, the opposite must be considered. Higher stress levels and longer sleep latency indicates that the individual may have difficulty falling asleep. Reminders that are targeted towards reducing the use of electronics and avoiding caffeine consumption after $5 \mathrm{pm}$ could be useful.

\subsection{Agreeableness}

Individuals who score high in agreeableness are associated with a longer sleep duration and may be more open to recommendations about how to further improve their sleep habits [18]. While those scoring low in agreeableness have not been linked to specific sleep behavior, these individuals are less cooperative and more competitive than those who score high for this characteristic [28].

Therefore, a person with a lower score in agreeableness could benefit from feedback that fuels their competitive nature. This idea could be leveraged through development of a gamification and community aspect in SleepHealth. Creating an online community within the app is one way for users to find motivation if they are less likely to respond purely to the individualized reminders. Ultimately, the gamification aspect would fuel an individual's competitive drive and promote positive sleep goals.

\subsection{Openness}

A high openness rating has been associated with longer sleep in one study, but there is still feedback that could improve users' sleep health [17]. A high score for openness indicates that the individual may be willing to try new suggestions that are outside of their current behavior patterns relating to sleep [28]. This would include suggestions about additional healthy behaviors, such as reducing caffeine intake and increasing exercise, in order to further improve sleep health. Additionally, individuals with a high score for openness may be open to suggestions about changing or improving their sleep goal if their sleep could be further optimized.

On the other hand, those scoring low in openness are much less likely to be willing to adopt any new ideas that SleepHealth suggests. Therefore, it is important to continue to reiterate true, factual, research supporting ideas that are directly related to sleep behavior. For example, this may include reminding the user about the stipulated number of hours of sleep that the National Sleep Foundation recommends in order to encourage the individual to reach that goal.

Additionally, other studies suggest that openness may not have as much of a direct correlation with sleep quality and quantity, but that it greatly affects social behavior [18]. This suggests that the aforementioned idea about gamification and community in SleepHealth could be very effective for those who are motivated by the social aspect of an app. 
Table 2. Design of feedback based on personality and chronotype characteristics

\begin{tabular}{|c|c|c|}
\hline Characteristic & Level & Planned Feedback (Reminders, Suggestions, Informational) \\
\hline \multirow[t]{2}{*}{ Conscientiousness } & Low & $\begin{array}{l}\text { - Reminders twice per week suggesting going to bed at a reasonable } \\
\text { time } \\
\text { - Reminder on Friday encourages maintaining the same sleep } \\
\text { schedule on weekends }\end{array}$ \\
\hline & High & $\begin{array}{l}\text { - Reminder on Monday of user's sleep goal for the week } \\
\text { - Message on Wednesday encourages avoidance of all-nighters and } \\
\text { caffeine consumption after 5pm }\end{array}$ \\
\hline \multirow[t]{2}{*}{ Neuroticism } & Low & $\begin{array}{l}\text { - No need for particular feedback or reminders based exclusively on } \\
\text { personality type }\end{array}$ \\
\hline & High & $\begin{array}{l}\text { - Reminders twice per week with suggestions and references about } \\
\text { how to control stress and improve mood }\end{array}$ \\
\hline \multirow[t]{2}{*}{ Extraversion } & Low & $\begin{array}{l}\text { - Message on Monday encourages avoidance of electronics before } \\
\text { bed and caffeine consumption after } 5 \mathrm{pm}\end{array}$ \\
\hline & High & $\begin{array}{l}\text { - Message on Friday about the detriment of an irregular weekend } \\
\text { sleep schedule with the suggestion to maintain the same sleep } \\
\text { schedule on weekends }\end{array}$ \\
\hline \multirow[t]{2}{*}{ Agreeableness } & Low & $\begin{array}{l}\text { - Message on Friday comparing user's sleep data that week to } \\
\text { national data or what the data should ideally look like for a college } \\
\text { student }\end{array}$ \\
\hline & High & $\begin{array}{l}\text { - No need for particular feedback or reminders based exclusively on } \\
\text { personality type }\end{array}$ \\
\hline \multirow[t]{2}{*}{ Openness } & Low & $\begin{array}{l}\text { Message on Monday provides factual statements to improve sleep } \\
\text { patterns, such as the National Sleep Foundation's recommendation } \\
\text { about number of sleep hours per night }\end{array}$ \\
\hline & High & $\begin{array}{l}\text { - Reminders twice per week suggesting going to bed at a reasonable } \\
\text { time } \\
\text { - Reminder on Friday encourages maintaining the same sleep } \\
\text { schedule on weekends }\end{array}$ \\
\hline \multirow[t]{2}{*}{ Chronotype } & Morning & $\begin{array}{l}\text { - Message on Monday encourages avoidance of electronics before } \\
\text { bed and caffeine consumption after } 5 \mathrm{pm}\end{array}$ \\
\hline & Evening & $\begin{array}{l}\text { Message on Monday with suggestion to set a reasonable bedtime } \\
\text { that will allow for } 8-10 \text { hours of sleep each night and with } \\
\text { information that sleepiness the day after an all-nighter or late night } \\
\text { will affect academics }\end{array}$ \\
\hline
\end{tabular}

\subsection{Chronotype}

In addition to personality traits, chronotype (i.e., preference toward morning or evening) will also be used to guide feedback.

Morning chronotypes may function better with early class times and have better academic performance because of their inclination towards working in the morning [29]. For these individuals, total sleep time is less of an issue and feedback for them will focus on how to improve sleep quality rather than sleep quantity.

For evening chronotypes, an emphasis on sleep quantity is more important since their later bedtimes encourage shorter total sleep time. 


\section{Conclusion and Future Work}

\subsection{Summary and Conclusion}

Sleep health is a critical issue for college students because of the potential for sleep deprivation to hijack their academic success. This paper proposes and describes the use of individual personality and chronotype characteristics to personalize feedback in a smartphone app as a means to engage users and promote behavioral change by accounting for their personal differences. These are concrete attributes that young adults possess and provide a basis for persuasive feedback. Our approach focuses on each of the steps of forming healthy sleep patterns through personalized reminders, altering unhealthy behaviors through individualized feedback, and reinforcing healthy behaviors through continual use of the app.

A smartphone app is an appropriate vehicle for feedback because most college students currently use smartphones. We anticipate that personalized feedback based on PSD and BCSS models and implemented in a smartphone app will improve their engagement and promote behavioral change through a unique personalized experience for each user.

\subsection{Planned Future Work}

Based on the design framework developed in this paper, we briefly present a concept prototype to demonstrate our planned enhancements to the existing SleepHealth app [3]. Specifically, in our future work, personality and chronotype measures and feedback dialogs will be fully integrated into SleepHealth. An example of this conceptual prototype with sample feedback messages is shown in Figure 1.

Feedback is designed generally to encourage maintenance of a reasonable bedtime throughout the week and to maintain a consistent sleep schedule on weekends. Personalization adapts this general feedback to address specific aspects of individual characteristics.

Figure 1 shows two sample feedback dialogs, one showing a reminder for an extraverted, eveningchronotype individual and the other for a highconsciousness, morning chronotype individual.

In the case of extraverted, evening chronotype individuals, feedback is designed to encourage maintenance of a reasonable bedtime throughout the week, but especially on weekends. Extraverted individuals tend to stay up much later on weekends, and this effect is exacerbated by a preference for evening activities. Thus, feedback focuses on misaligned weekend sleep patterns that could disrupt users' sleep patterns during the subsequent week.
In the case of the high-conscientiousness, morning chronotype user, feedback is focused towards reinforcing the user's sleep goal, thereby leveraging the goal-driven nature of a conscientious person. The suggestion to not consume caffeine after $5 \mathrm{pm}$ is particularly applicable to morning chronotypes because of the potential for these individuals to use caffeine to stave off sleepiness later in the day. The same is true for electronics before sleeping, which can also worsen sleep quality. Since highly conscientious users tend to be intrinsically motivated to reach their sleep goal length, the focus of feedback (as an external motivator) is on improving sleep quality.
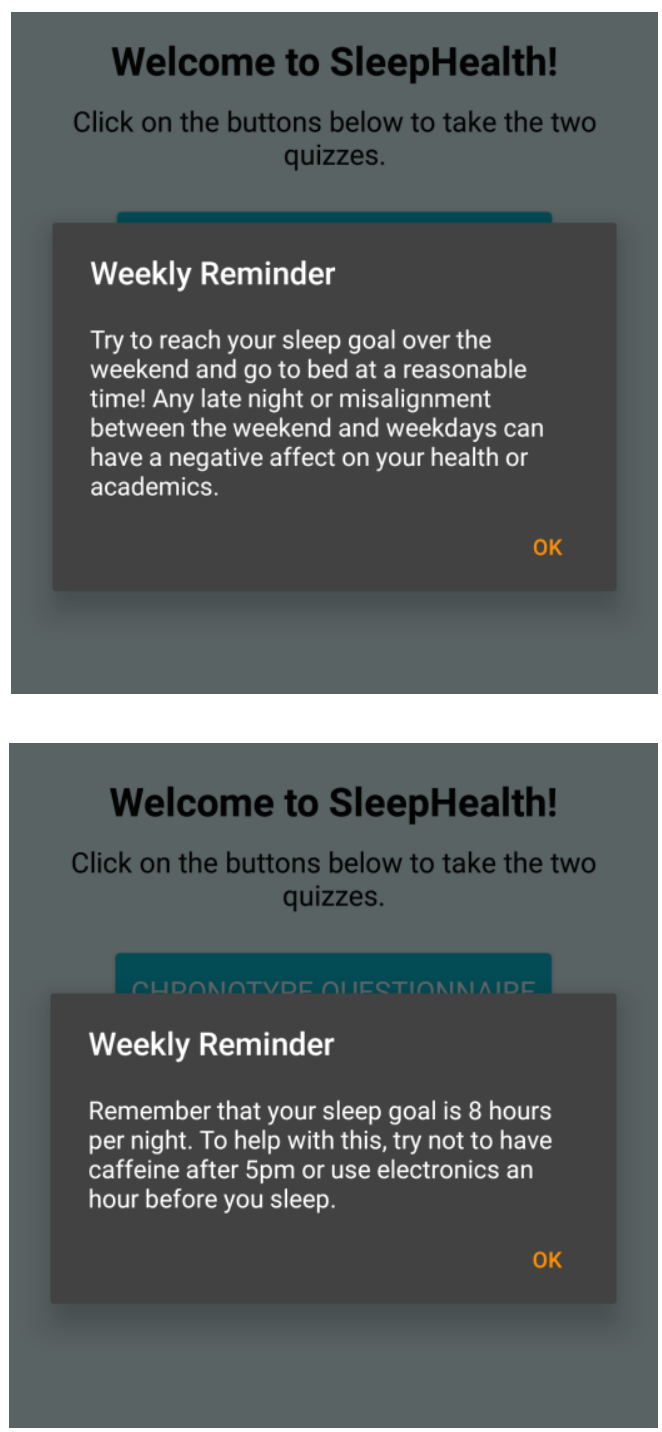

Figure 1. Sample feedback personalized for a high extraversion, evening-chronotype user (top) and for a high conscientiousness, morning-chronotype user (bottom) 
A formal user study will then be conducted to evaluate the usability, functionality, helpfulness, and overall success of the enhanced SleepHealth app. This will provide empirical results about how well the personalization works and what may need to be revised in order to increase the effectiveness and user experience for users.

\section{Acknowledgements}

The first two authors acknowledge the support of a Clare Boothe Luce Foundation Research Award which made this research possible.

\section{References}

1. Taylor, D.J., et al., The Role of Sleep in Predicting College Academic Performance: Is It A Unique Predictor? . Behavioral Sleep Medicine, 2013. 11(3): p. 159-172.

2. Hershner, S.D. and R.D. Chervin, Causes and consequences of sleepiness among college students. Nature and Science of Sleep, 2014. 6: p. 73-84.

3. Djamasbi, S., et al., Designing and Testing UserCentric Systems with both User Experience and Design Science Research Principles. The 22nd Americas Conference on Information Systems (AMCIS 2016), 2016.

4. Pearson. Pearson student mobile device survey 2015. 2015; Available from: http://www.pearsoned.com/wpcontent/uploads/2015-Pearson-Student-Mobile-DeviceSurvey-College.pdf.

5. Wilson, E.V., et al., Using a key informant focus group, formative user testing, and theory to guide design of a sleep health BCSS, in Proceedings of the 50th Hawaiian International Conference on System Sciences. 2017: Waikoloa Village, HI.

6. Oinas-Kukkonen, H. and M. Harjumaa, Persuasive systems design: Key issues, process model, and system features. Communications of the Association for Information Systems,, 2009. 24(28): p. 486-500.

7. Duggan, K.A., et al., Personality and healthy sleep: the importance of conscientiousness and neuroticism. Public Library of Science One, 2014. 9(3): p. e90628.

8. Langrial, S., H. Oinas-Kukkonen, and S. Wang, Design of a web-based information system for sleep deprivation-A trial study, in International Conference on Well-Being in the Information Society. 2012, Springer Berlin Heidelberg. p. 41-51.
9. Langrial, S., et al. Influence of persuasive reminders and virtual rehearsal on information systems for sleep deprivation. in 2014 PACIS Proceedings. 2014.

10. Foundation, N.S. Teens and Sleep. National Sleep Foundation [Web] 2016 June 2017]; Available from: https://sleepfoundation.org/sleep-topics/teens-and-sleep.

11. Herman, R. How Lack of Sleep Affects Cholesterol. Sleep Health [Web] 2016 June 2017]; Available from: https://www.sleepassociation.org/2016/04/24/lack-sleepaffects-cholesterol/.

12. Onyper, S.V., et al., Class start times, sleep, and academic performance in college: a path analysis. Chronobiology International: Journal of Biological and Medical Rhythm Research, 2012. 29(3): p. 318-35.

13. DeMartini, K.S. and L.M. Fucito, Variations in Sleep Characteristics and Sleep-Related Impairment in AtRisk College Drinkers: A Latent Profile Analysis. Healthy Psychology, 2014. 33(10): p. 1164-1173.

14. Chennaoui, M., et al., Sleep and exercise: a reciprocal issue? Sleep Medicine Reviews, 2015. 20: p. 5972.

15. Taylor, D.J. and A.D. Bramoweth, Patterns and consequences of inadequate sleep in college students: substance use and motor vehicle accidents. J Adolesc Health, 2010. 46(6): p. 583-596.

16. Champlin, S.E., K.E. Pasch, and C.L. Perry, Is the Consumption of Energy Drinks Associated With Academic Achievement Among College Students? Journal of Primary Prevention, 2016. 37(4): p. 345-359.

17. Williams, P.G. and T.L. Moroz, Personality vulnerability to stress-related sleep disruption: Pathways to adverse mental and physical health outcomes. Personality and Individual Differences, 2009. 46(5-6): p. 598-603.

18. Hintsanen, M., et al., Five-Factor Personality Traits and Sleep: Evidence From Two Population-Based Cohort Studies. Health Psychology, 2014. 33(10): p. 1214-1223.

19. Oinas-Kukkonen, H., Behavior change support systems: A research model and agenda, in Persuasive Technology. 2010, Springer Berlin Heidelberg. p. 4-14.

20. Oinas-Kukkonen, H., A foundation for the study of behavior change support systems. Personal and Ubiquitous Computing, 2013. 17(6): p. 1223-1235.

21. Bem, D.J., Self-perception: An alternative interpretation of cognitive dissonance phenomena. Psychological review, 1967. 74(3): p. 183-200. 
22. Truity. The Big Five Personality Test. 201602 August 2016]; Available from: http://www.truity.com/test/big-five-personality-test

23. Costa Jr., P.T. and R.R. McCrae, Four ways five factors are basic. Personality and individual differences, 1991. 13(6): p. 653-665.

24. Hahn, E., J. Gottschling, and F.M. Spinath, Short measurements of personality - Validity and reliability of the GSOEP Big Five Inventory (BFI-S). Journal of Research in Personality 2012. 46(3): p. 355-359.

25. Smith, C.S., C. Reilly, and K. Midkiff, Evaluation of three circadian rhythm questionnaires with suggestions for an improved measure of morningness. Journal of Applied Psychology, 1989. 74(5): p. 728-738.

26. Randler, C., Validation of the full and reduced Composite Scale of Morningness. Biological Rhythm Research, 2008. 40(5): p. 413-423.

27. Hervé Caci, A.A., Philip Bohle, Vincenzo Natale, Chanthika Pornpitakpan, Andrew Tilley, Transcultural Properties of the Composite Scale of Morningness: The Relevance of the "Morning Affect" Factor. Chronobiology International, 2005. 22(3): p. 523-540.

28. Cherry, K. What Are the Big 5 Personality Traits? [Web] May 2017 June 2017]; Available from: https://www.verywell.com/the-big-five-personalitydimensions-2795422.

29. Randler, C., Morningness-eveningness, sleep-wake variables and big five personality factors. Personality and Individual Differences, 2008. 45(2): p. 191-196. 